\title{
Corela
}

Cognition, représentation, langage

HS-30 | 2020

Phonétique, littérature et enseignement du FLE :

théories et recherches

\section{L'intérêt de l'utilisation du support dramatique : un outil pour entendre et écouter, percevoir et comprendre, parler et s'exprimer en langues étrangères}

Fanny Auzéau

\section{OpenEdition \\ Journals}

Édition électronique

URL : http://journals.openedition.org/corela/10254

DOI : 10.4000/corela. 10254

ISSN : 1638-573X

Éditeur

Cercle linguistique du Centre et de l'Ouest - CerLICO

Référence électronique

Fanny Auzéau, «L'intérêt de l'utilisation du support dramatique : un outil pour entendre et écouter, percevoir et comprendre, parler et s'exprimer en langues étrangères », Corela [En ligne], HS-30 | 2020, mis en ligne le 20 avril 2020, consulté le 23 mai 2020. URL : http://journals.openedition.org/corela/ 10254 ; DOI : https://doi.org/10.4000/corela.10254

Ce document a été généré automatiquement le 23 mai 2020

\section{cc) (i) (3)}

Corela - cognition, représentation, langage est mis à disposition selon les termes de la licence Creative Commons Attribution - Pas d'Utilisation Commerciale - Partage dans les Mêmes Conditions 4.0 International. 


\title{
L'intérêt de l'utilisation du support dramatique : un outil pour entendre et écouter, percevoir et comprendre, parler et s'exprimer en langues étrangères
}

\author{
Fanny Auzéau
}

1 Cet article a pour objectif de croiser la littérature des différents domaines impliqués dans l'enseignement de la prononciation qui font du support de théâtre un input riche tant sur le plan de la production que de la perception. Dans cet article, nous appuierons notre propos sur des expérimentations menées dans le cadre universitaire, mais ne souhaitons pas en décrire les dispositifs pratiques (cf. Auzéau, $2018: 163-165)$. Les extraits littéraires dans notre contexte des saynètes ou scènes de pièces de théâtre sont souvent une entrée en matière dans la langue. Ces supports, parfois authentiques ou didactisés, mais nous n'entrerons pas dans ce débat, permettent d'appréhender des faits de langue à différents niveaux (sémantique, syntaxique, lexical, morphologique, phonologique, phonétique, graphique). Cette introduction à la langue-culture nous intéresse à différents points de vue, tout d'abord perceptif, mais aussi sensitif et productif car en interprétant le texte d'un tiers, d'un auteur ou en produisant soimême ce type de support. Comme le décrit Cyrulnik (2000:44) : «pour apprendre une langue, il ne faut pas seulement assimiler les sons, les mots, les règles, il faut acquérir la manière d'y traduire ses sentiments ». Au cœur de l'extrait littéraire, nous retrouvons à la fois un message linguistique, de l'implicite culturel et une approche sensorielle guidée par les didascalies ou l'interprétation de l'acteur. L'implicite culturel et interculturel et la sensorialité font partie de cet objet littéraire. L'apprentissage d'un nouveau système phonétique-phonologique n'est pas une tâche aisée (Orion, 2012), car il demande une maitrise physiologique, une perception d'un nouvel échantillon sonore segmental et suprasegmental, mais également une pratique régulière. Cela signifie que l'étude d'un phonème doit rapidement être contextualisé, confronté à des usages 
multiples pour que les caractéristiques d'un son à l'isolé deviennent spontanées, au même titre que le fait l'enfant dans sa langue maternelle. Comprendre une langue étrangère ne signifie pas seulement entendre des sons, des phonèmes, distinguer des oppositions ou trouver similitudes entre eux, mais également de "[s]'approprier la prononciation d'une nouvelle langue [ce qui] relève d'un processus impliquant une nouvelle conscience linguistique, physique et identitaire plurielle, ce qui amène l'apprenant à développer son acuité auditive » (Rolland, 2011 : 73) ; l'apprenant devra être prêt à ces changements pour s'ouvrir à ce nouveau spectre sonore. Cette nécessité d'implication, d'engagement dans l'acte d'enseignement/apprentissage de la prononciation transforme le processus biologique d'écoute en une activité volontaire. Toutefois, il ne faut pas perdre de vue que ces textes sont des œuvres artistiques qui permettent de (re)voir les objets du réel, d'interpréter des situations, des sentiments et/ou des attitudes. Cette création permet à l'apprenant de s'approprier un peu plus l'univers de la langue cible.

\section{Le texte de théâtre}

2 Le texte de théâtre a pour intérêt principal d'être le reflet d'une forme parlée de la langue étrangère car les prédispositions musicale et rythmique sont à la source de cette création. Celui-ci se situe à un carrefour de disciplines, d'un côté il « appartient à la littérature par ses œuvres dramatiques, [d'un autre côté] au spectacle par ses techniques de jeu et de la scène, à l'histoire par ses rites et ses traditions » (David, $2012: 5)$. Ce support a une résonance particulière par sa nature, les indications qu'il comporte, mais également par la polyphonie qui le caractérise, car «[1]e texte [théâtral] s'adresse à l'auteur, au metteur en scène, à l'acteur, au spectateur » (Parisse, 2011 : 117), c'est ce qui rend sa lecture parfois difficile aux non-initiés, car «le texte théâtral prend son véritable relief lorsque le lecteur assiste à une mise en scène du texte » (ibid:119). En langues étrangères, cette lecture n'est pas moins aisée, car elle nécessite un nouveau décodage. Ce mouvement de va-et-vient entre l'écrit et l'oral est constant dans ce support : l'écrit reproduit un dialogisme oral ; la production orale doit paraître véritable grâce au jeu d'acteur alors qu'elle émane d'une production écrite. L'acte de mise en voix du texte dramatique est un exercice complexe à différents niveaux. Cette difficulté est mise en lumière par Kerbrat-Orecchioni $(1984: 47)$ qui précise que le «dire» est automatiquement le «faire» car la «parole dramatique est tout entière mise au service de l'action, et réciproquement, c'est par le biais du langage que tout advient ». La figure proposée par cette linguiste met en avant l'emboîtement à différents niveaux de la communication dans l'art de la scène, mais également que « ces choses dites à mots couverts, ces arrière-pensées sous-entendues entre les lignes pèsent lourd dans les énoncés » (ibid : 6). C'est pourquoi il est possible d'utiliser ce type de support avec des niveaux intermédiaires à avancés (B1+ à C2). La difficulté dans la pratique théâtrale et son emprunt dans l'enseignement repose donc sur le fait que cette retranscription de la réalité ne soit pas une simple lecture. Son utilisation ne doit pas se limiter à «la lecture à haute voix » ou à une "transmission vocale du texte » (Bajard, 2002 : 221), mais bien comme un support de « théâtralisation » (Aden, $2007: 68$ ).

3 Fónagy (1991:320) décrit ainsi l'oralité présente dans l'acte de création littéraire : « Le poète quand il écrit son poème, a généralement déjà une mélodie, un rythme, il cherche un texte, il construit son texte à partir d'une mélodie, d'une vision mélodique de 
l'ensemble", ce que nous pouvons transposer au texte dramatique. Le dramaturge a une idée esthétique, scénique et parfois graphique de son œuvre en cours de création. En s'appuyant sur un tel support l'apprenant est amené à parler, à s'exprimer et à communiquer avec ses pairs en s'appropriant le texte d'un tiers. Cette caractéristique peut représenter un obstacle pour l'étudiant praticien de par sa culture, son caractère et son ressenti dans le groupe, mais cet objet littéraire devient un support didactique intéressant sur de nombreux points.

\section{a) Entendre et écouter}

Il est courant de lire dans la littérature scientifique que la perception est généralement la première étape à l'appropriation d'un nouveau système sonore (Guimbretière, 1994). Toutefois, le traitement de la matière sonore ou le décryptage du "paysage sonore " (Llorca, 1985) dépend du degré d'implication du destinataire. Entendre est une activité arbitraire, dépendante de nos capacités physiques et biologiques, tandis qu'écouter est une activité intentionnelle qui nécessite une motivation, un processus volontaire de la part du destinataire. Ainsi, "l'intentionnalité est le lien unifiant qui fait que l'expérience de la conscience n'est pas un ramassis d'impressions et d'autres phénomènes, mais un processus unitaire, doué de sens" (Patocka, 1992:86); ceci appuie la thèse d'un contrat d'apprentissage tacite ou non entre l'apprenant et l'enseignant. Cette intentionnalité est d'autant plus importante dans la formation pour adultes. L'écoute, au même titre que la compréhension, sont objectifs tandis qu'entendre est subjectif et abstrait. Cependant, comme le relativise Matter (2006:22), ce n'est pas l'unique raison «[t]out maître de langue sait également qu'une bonne perception auditive, si elle est une condition nécessaire pour aider l'apprenant à atteindre son niveau maximum, n'est apparemment pas une condition suffisante, puisque même un apprenant ayant une prononciation peu authentique, est capable de comparer la prononciation du maître non-natif avec la voix du modèle natif ». Dans cette citation, nous constatons que l'écoute de la parole peut permettre une catégorisation (faible ou forte) d'une différence de production, sans assurer pour autant une production la plus proche de la «norme » escomptée. Il semble normal que nous y soyons habitué dans notre langue maternelle, car c'est «l'une de nos activités les plus fréquentes» (Durand, 2002:5). Ceci nous renvoie au concept décrit par Meschonnic (1982:18) : «L'oralité » ne se limite pas à traiter de la matière phonique, en réception comme en production, mais est « l'ensemble des faits et processus liés à une parole communautaire qui : a) se déploie dans des contextes ritualisés ; b) s'inscrit dans la répétition (la récitation, la charité) et réfère à une mémoire identitaire; c) met en jeu la voix et le corps portés par une diction spécifique; d) repose sur un mode d'énonciation gnomique mettant en œuvre, dans le cadre d'une tradition orale. ». Maîtriser la matière sonore d'une langue étrangère nécessitera des aptitudes variées, des connaissances théoriques et pratiques ainsi qu'un entrainement ou une confrontation régulière avec la langue cible, comme l'enfant le fait en langue maternelle.

5 Traditionnellement dans l'enseignement de la prononciation, les paires minimales ou des mots à l'isolé servent d'input de sensibilisation aux traits phonétiques/ phonologiques, mais, dans le cas de la sensibilisation par un extrait d'une pièce de théâtre, cela sera plus délicat. Un second degré d'analyse devra être activé : l'analyse 
des insinuations et des sous-entendus. Recanati $(1981: 141)$ mentionne cette pluralité, car ce genre dramatique peut " laisser entendre, donner à entendre et sous-entendre ", ce qui a une incidence directe sur la nature de la communication symétrique (entre les acteurs « réels ») ou asymétrique (entre l'auteur et l'acteur). Le texte théâtral n'est pas seulement le mariage d'un texte et d'une voix, mais la fusion de plusieurs codes (langagiers, scéniques, artistiques) (Benintendi-Simond, 2011:102). Certes, les didascalies apportent une aide à la lecture, mais peuvent également guider la mise en scène. Elles ne sont, toutefois, pas des «injonctions faites par l'auteur au praticien de théâtre » (Ailloud-Nicolas, $2011: 85$ ), mais un balisage qu'il peut à sa guise suivre. C'est ainsi que le résume Régy (2002: 25) : l'acteur présente « ce que le texte fait voir ». Ngo Mbai $(2011: 132)$ précise même que le théâtre est « un genre littéraire complet qui donne à lire, à entendre et à voir ». C'est un support en relief qui « porte la labialité du texte littéraire, la parole scénique» (Mouratidou, 2009 : 249). Les passages de Pour un oui ou pour un non (1982) de Nathalie Sarraute sont emplis d'oralité, de marques d'hésitation qui font partie des compétences intonatives, généralement classées comme phonétique-phonologiques dans les grilles d'évaluation. Un autre exemple, tout aussi éclairant, est la scène d'ouverture de la pièce de Yasmina Reza : Art (1994), qui favorise également l'expression de l'étonnement et de la colère ( $c f$. l'extrait cité par Abry et al., $2008: 48)$. Au sens littéral, cela ne fait pas uniquement référence à la phonétique ou à la phonologie, mais en pratique, il faut s'appuyer sur chaque consonne, prendre garde aux différents traits distinctifs, respecter le découpage syllabique et la courbe intonative pour que le texte prenne corps, que les mots sonnent et résonnent avec sens. Cela revient donc à activer les connaissances et compétences phonétiques/phonologiques de l'apprenant dans la langue cible.

6 Dans son usage pédagogique, l'apprenant sera en mesure d'entendre un échantillon de langue sélectionné par l'enseignant, mais d'écouter ses partenaires pour faire vivre le dialogue. L'entente entre les interactants ne se limite pas à l'audition, mais bien à une attention globale aux éléments paraverbaux, à la proprioception, aux gestes et aux mouvements, ainsi qu'à l'espace scénique. Nous voyons ainsi que cette pratique ne se cantonne pas à une récitation ou à une reproduction, mais bien à une approche globale des éléments constitutifs des échanges. Le silence est également un élément significatif à cette exposition à la langue cible. Le Meur (2012) le définit très justement: «Le silence de la parole n'est pas l'actio, car il agit aussi bien dans l'actio que dans la parole. Le silence n'est pas silence de l'action, il est le silence dans l'action silencieuse ».

7 Le texte dramatique est donc un support qui extrait des formes verbales contextualisées et assemblées pour répondre à un dialogisme nécessaire à la représentation théâtrale. L'apprenant pourra ainsi y retrouver des formes entendues, connues, mais également maîtriser un enchaînement de phonèmes, de syntagmes et d'idées. L'apport du support ne se limite pas à la sphère vocale, mais également, du point de vue sociolinguistique, à l'adaptation au contexte.

\section{b) Percevoir et comprendre}

8 Ces deux actions peuvent également s'appréhender au sens réflexif se percevoir pour se faire comprendre, car le comportement communicatif est aussi une manière de: « donner sa parole, c'est lier et se lier» (Fabre et al. $2005: 6$ ). Les éléments verbaux ne sont pas les seuls indicateurs. Les subtilités de la langue sont aussi perceptibles à 
travers les gestes, la gestuelle et les indices corporels. Selon Aden (2006:38), il est nécessaire de " percevoir avec tous ses sens, l'ouïe, le regard, le toucher, à décoder et organiser le perçu et lui donner du sens pour pouvoir agir ». C'est pourquoi des tâches de compréhension globale (à partir de support écrit ou oral) ne sont pas l'unique entrée en matière dans un texte dramatique. Detey et Racine $(2016: 87)$ précisent que «l'effet d'instruction est meilleur lorsque celle-ci est de type-"focus-sur-la-forme" (intégration du travail phonétique à des activités communicatives) plutôt que "focussur-les-formes" (travail uniquement sur les formes phonétiques) affectant non seulement la précision phonétique mais aussi la fluence». Le contexte apporte des éléments majeurs et signifiants au texte en lui-même, nous retrouvons au travers de cette problématique, la méthodologie «focus on meaning» (Ellis, 2001). Dans le texte théâtral, les traits phonétiques/phonologiques ou les contours prosodiques rencontrés sont multiples car ils représentent un échantillonnage de la langue en contexte. Contrairement aux exercices généralement proposés comme input à l'analyse d'un fait phonétique, phonologique ou prosodique, il ne s'agit pas de skills ou paires minimales sélectionnés pour leurs caractéristiques sonores, mais bien significatives. Le texte théâtral, comme toute œuvre littéraire, doit avoir du sens pour faire sens.

9 Les didascalies peuvent mettre en lumière certaines interprétations, mais le sensible et l'appropriation du texte seront personnel pour, par la suite, l'échanger avec un ou des partenaire(s) de jeu. S'exprimer nécessite un engagement de l'émetteur au destinataire et une coopération, sans négliger les éléments extérieurs (décor, personnages sur scène...) qui ont une importance aussi significative, d'autant plus, lorsque le texte théâtral est utilisé dans le cadre d'un enseignement par les techniques dramatiques. Toutefois, comme le relève Alazard et al. (2011:50), « le problème est que le véritable oral ne se retrouve jamais à l'écrit. Quelle que soit la transcription, elle est impuissante à rendre toute la richesse qui se dévoile spontanément à l'oral : l'intonation, le rythme, les silences, les changements de débit, les variations de tempo, le timbre, la tonicité, les indices sociaux ou affectif, tout ce que la voix transmet et qui ne s'écrit pas ». Des essais, des tentatives, des interprétations variées et variables seront à mettre en place, à favoriser et à valoriser dans l'enseignement/apprentissage d'une langue qu'elle soit considérée comme maternelle ou étrangère. D'autant plus que la première phase de découverte d'un texte se fait généralement par une tâche de lecture, ce qui représente des difficultés supplémentaires pour un apprenant en langue étrangère, car « il doit non seulement décoder la chaîne graphique et la recoder en chaîne phonémique, mais aussi organiser simultanément le matériau linguistique en groupes sémantiquement et syntaxiquement cohérents via des patrons prosodiques de la L2» (ibid. : 50). Cela peut entraîner un "ralentissement dans l'encodage de la chaîne parlée ainsi qu'une incapacité à actualiser les phonèmes dans les limites de prononciation acceptables » (ibid. : 50) entravant la compréhension.

Dans l'enseignement/apprentissage des langues étrangères, le support textuel et l'introduction de l'écrit sont délicats aux niveaux A1 et A2 (élémentaires), la transposition graphique peut induire en erreur la production orale et ralentit le débit de parole. Le français, comme l'anglais, ne sont pas des langues au rapport graphiephonie simple comme en italien ou en allemand. Les activités introductives à l'interprétation orale de texte passent généralement par une phase écrite qui seront plus significatives à des niveaux supérieurs (B1, B2 et $\mathrm{C} 1)$, comme le préconisait déjà Guberina (1954) avec la méthode verbo-tonale. Car l'aptitude langagière nécessite la 
maîtrise de nombreux éléments: l'interprétation, l'intention et le ton donnés par l'enseignant ou l'intervenant en pratique théâtrale seront des facilitateurs de la compréhension. C'est pourquoi Aden (2009:174) conclut que «maîtriser de mieux en mieux une langue c'est devenir de plus en plus créatif dans cette langue, l'un n'étant pas la conséquence de l'autre, mais les deux se renforçant dans une boucle rétroactive ". La création se retrouve dans des actions ponctuelles (comme dans l'improvisation par exemple), mais également dans la production de textes dramatiques ou de scénarii. Ce concept peut également être interprété comme une production, une proposition de texte théâtral, ce qui représente un excellent moyen pour reproduire les codes du théâtre (dialogisme, forme d'adresse, didascalie...), mais également de devenir auteur de sa propre création.

\section{c) Parler et s'exprimer}

11 Parler et s'exprimer grâce aux textes théâtraux ne signifient pas uniquement vocaliser, prononcer des mots choisis par un tiers, mais surtout interpréter un rôle ou chercher à en jouer un autre. Cela signifie à la fois prendre des risques (linguistiques, corporels et interprétatifs), mais aussi accepter le regard des autres sur ce qu'on représente. La répartition entre les contours gestuels et phoniques est la suivant : « les deux tiers de la communication sont portés par le non-verbal et seulement un tiers par le verbal» (Lazaraton, $2004: 81$ ). Dans ce contexte, les apprenants changent aussi leur statut dans la classe. Ils doivent être capables de maintenir une distance entre fiction et réalité, de comprendre que parler sur scène comporte des pratiques et techniques propres aux planches, que l'espace de liberté proposé lors des activités d'improvisation ou d'interprétation sont des occasions d'expérimenter, de tester et parfois de se mettre en danger.

L'outil principal est la voix, qui nécessite une maîtrise de la colonne d'air, une adaptation à la vocalisation scénique pour s'adresser au plus grand nombre et manier la qualité vocale de son instrument qu'est la voix. Maîtriser l'appareil phonatoire est essentiel dans la production dramatique, et comme le mentionne Brown (1994) «la pose de voix (voice setting)» est également un excellent point de départ pour la formation à la prononciation en L2. Jones et Evans $(1995: 245-246)$ nomment trois raisons supplémentaires à cette technique de « la pose de voix » comme point de départ dans le travail de la prononciation: premièrement, il constitue une approche plus holistique dans laquelle, dès le début, différents éléments de prononciation sont vus comme intégrés. Deuxièmement, il donne aux étudiants une chance d'éprouver la prononciation aux niveaux intuitifs et communicatifs avant de passer à une exploration plus analytique des éléments spécifiques de la phonologie. Enfin, le travail de la qualité de la voix aide les étudiants à améliorer leur image quand ils parlent anglais [ou une langue étrangère], et augmente ainsi leur confiance. Vocaliser apporte une " motivation instrumentale [qui] est donc pragmatique et n'a pas besoin d'être fondée dans la langue : on a besoin de la langue pour atteindre un but qui se trouve en dehors de la langue" (Mater, 2006:28). C'est en cela que susciter la parole, favoriser le dialogue et donc l'interaction sont des formes de stimuli à reproduire la réalité dans un lieu neutre, un espace d'échange et d'essais. Cette prise de conscience et de confiance dans un terrain respectueux et bienveillant est un premier pas vers l'autonomie hors de la classe. 

doit « renoncer à une position de toute puissance » (Page, $2000: 137)$, bien qu'il soit la clef de voûte du cours, il doit moduler ses interventions en fonction de la réception du groupe. Laisser place à l'inconnu et au hasard n'est pas chose aisée dans un schéma d'enseignement/apprentissage dit " classique ", mais dans l'utilisation des supports et donc des disciplines artistiques le défi sera tout autre et les dispositions de l'enseignant également. Cormanski $(2005: 10)$ en détaille les contours : « [s]on rôle essentiel est celui d'un guide chargé d'agencer des "situations à communiquer", de fournir aux apprenants qui vont les mettre en acte et se trouver alors le besoin de dire, la matière langue dans son aspect structurel et fonctionnel. Selon les activités proposées, il aura parfois le rôle de "souffleur", discrètement positionné derrière l'apprenant en action, pour alimenter verbalement l'interaction qui se construit ", lorsque la scène est jouée et que le texte dramatique prend vie, mais également d'animateur, de metteur en scène, en voix et en espace. d'apprentissage des usages oraux d'une langue dans une culture donnée » (Mégévand, 2001:39) car l'utilisation d'un support dramatique permet que "l'on décentre l'attention de l'apprenant de la dimension linguistique (sans pour autant la perdre de vue puisque c'est un cours de langue) pour la focaliser temporairement sur le support créatif» (Puozzo, 2013:37). S'approprier le texte théâtral est prétexte à la manipulation de la matière sonore, en entrant dans la peau d'un autre, l'étudiant/ acteur emploie une identité vocale étrangère grâce à laquelle il devra s'approcher d'une réalité. Dans ce type de pratique, la notion de performance ne s'entend pas au niveau de la production vocale mais de l'interprétation verbale (phonétique, phonologique et intonative) et paraverbale. L'authenticité du faire-semblant ne devra pas tomber dans la caricature pour autant.

De nombreuses expériences sont menées sur le terrain, l'apprentissage " par corps » est une pratique désormais courante. Lors d'une expérience menée au cours d'un semestre à l'Université dans le cadre d'un cours de prononciation, nous avons constaté que la cohésion du groupe était un atout fondamental pour la prise de parole en public. Grâce à de nombreuses activités dramatiques, de situations fictives, la parole se déliait et l'aisance en langue étrangère était visible. Ces activités libres de supports étaient au début vécues comme une prise de risque majeure, mais, plus les semaines passaient, plus le dialogue entre les interactants se formait. Lorsque nous utilisions un support papier écrit par un tiers, la position corporelle et l'attention étaient tout d'abord centrées sur la performance, au sens d'agir de manière adéquate et non d'action adaptée à la situation. Le passage à la mémorisation du texte a permis aux apprentisacteurs de se découvrir comme porte-parole du texte d'un tiers et acteur dans une langue étrangère. Les données collectées lors de cette expérimentation étaient centrées sur le niveau segmental, mais il aurait été intéressant d'observer le niveau suprasegmental qui nous semble avoir évolué de manière significative (Auzéau, 2018 : 157-167).

Au cours de cet article, nous avons souhaité montrer les nombreuses fonctions du texte dramatique dans le jeu théâtral, car il porte des caractéristiques variées de la communication multimodale et réactive toutes les composantes de phonétique, phonologie et des faits suprasegmentaux, très souvent délaissés. La didactisation de ces supports présente dans de nombreux ouvrages en propose une exploitation bi- 
dimensionnelle (je comprends puis je répète), mais nous proposons une troisième dimension à ce support qui est "j'interprète " pour mener à l'individualisation de la parole et donc à un «je m'exprime». L'apprenant s'approprie les catégorisations, les traits distinctifs pour produire. Cette multimodalité permet d'entrevoir la complexité de la maîtrise d'une langue étrangère : ses codes ne sont pas uniquement retranscrits, mais également implicites, tant sur le plan de l'usage que de la signification. S'approprier ce support relève d'une maîtrise élevée des compétences langagières et des aptitudes communicatives, c'est pourquoi les niveaux B et C du Cadre européen commun de référence pour les langues semblent les plus appropriés pour les appréhender. La fluidité de la parole et la base de connaissance lexicale, grammaticale et syntaxique, mais également le temps de confrontation à la langue cible sont des atouts nonnégligeables à l'utilisation du support de théâtre. Comme le clarifie Ryngaert (1996:74) : «On joue autant pour comprendre que pour se faire comprendre, ce qui oblige à faire face simultanément à l'élaboration et à la communication d'un message ». C'est à notre sens, l'essence même de l'enseignement-apprentissage d'une langue étrangère.

\section{BIBLIOGRAPHIE}

ABRY D., DAÂS Y., DESCHAMPS H., FERT C., RICHAUD F. et SPERANDIO C., 2008, Ici 2 (niveau A2), Paris, CLE International, coll. « Ici ».

ADEN J., 2006, « To play, to act... perchance to learn ? », Les Cahiers pédagogiques, n 448, p. 38-39.

-, 2007, Construction identitaire et altérité en didactique des langues, Paris, Éditions Le Manuscrit.

-, 2009, « La créativité artistique à l'école : refonder l'acte d'apprendre », Synergies Europe, $\mathrm{n}^{\circ} 4$,

p. 173-180.

AILlOUD-NICOLAS C., s. d., « Un enseignement de la dramaturgie est-il possible dans les classes de collège et de lycée ? ». Disponible sur : <http://www.gerflint.fr/Base/France8/Ailloud-

Nicolas.pdf>.

ALAZARD C., ASTÉSANO C., BILLIÈRES M. et ESPESSER R., 2011, « Rôle de la prosodie dans la structuration du discours : proposition d'une méthodologie d'enseignement de l'oral vers l'écrit en Français Langue Étrangère ", in H.-Y. YOO et E. DELAIS-ROUSSARIE (dir.), Actes d'IDP 2009, Paris, septembre 2009, ISSN 2114-7612, p. 49-61. Disponible sur : <http://makino.linguist.jussieu.fr/idp09/docs/ IDP_actes/Articles/alazard.pdf>.

AUZÉAU F., 2018, « Résumé de thèse : Pertinences de l'enseignement de la prononciation par le théâtre et par le chant », dans V. LAURENS et L. NICOLAS (dir.), « Le français à visée professionnelle : recherches et dispositifs de formation. Actes des $57^{\mathrm{e}}$ et $58^{\mathrm{e}}$ rencontres $»$, Les Cahiers de l'ASDIFLE, Paris, CLE International, p. 157-167.

BAJARD É., 2002, Caminhos da escrita : espaços de aprendizagem, São Paulo, Cortez Editora.

BENINTENDI-SIMOND M., 2011, « Langues, cultures, théâtres, quelles alliances ? De l'apprentissage des langages par la pratique théâtrale », Synergies France, nº 8, p. 101-104. 
BROWN H. D., 1994, Teaching by Principles : An Interactive Approach to Language Pedagogy, Englewood Cliffs, N.J., Prentice Hall Regents.

CORMANSKI A., 2005, Techniques dramatiques : activités d'expression orale, Paris, Hachette, coll. «Pratiques de classe ».

CYRULNIK B., 2000, Les nourritures affectives, Paris, Odile Jacob.

DAVID M., 2012, Le théâtre, Paris, Belin.

DETEY S., RACINE I., KAWAGUCHI Y., et EYCHENNE J., 2016, La prononciation du français dans le monde : du natif à l'apprenant, Paris, CLE International, coll. « Référence ».

DURAND P., 2002, La phonétique du F.L.E. au niveau avancé, publication du laboratoire de Phonétique et F.L.E, Université de Provence. Disponible sur : <http://www.lpl-aix.fr/ fulltext/1909.pdf>.

ELLIS R., 2001, «Introduction : Investigating Form-Focused Instruction », Language Learning, $\mathrm{n}^{\mathrm{0}}$ 51(1), p. 1-46.

FABRE H. et SAEZ G., 2005, « Dossier de presse du colloque national "Corps et voix", Culture, éducation et formation », Institut Universitaire de Formation des Maîtres, Académie de Montpellier.

FÓNAGY I., 1991, La vive voix : essais de psycho-phonétique, Paris, Payot.

GUBERINA P., 1954, Valeur logique et valeur stylistique des propositions complexes : théorie générale et application au français, Zagreb, Epoha.

GUIMBRETIÈRE É., 1994, Phonétique et enseignement de l'oral, Paris, Didier Hatier.

JONES R. H. et EVANS S., 1995, « Teaching pronunciation through voice quality », ELT Journal, vol. 49, $\mathrm{n}^{\circ} 3$, p. 244-251.

KERBRAT-ORECCHIONI C., 1984, « Pour une approche pragmatique du dialogue théâtral », Pratiques. Linguistique, littérature, didactique, $\mathrm{n}^{\circ} 41$, p. 46-62.

-, 1986, L'implicite, Paris, Armand Colin.

LAZARATON A., 2004, « Gesture and Speech in the Vocabulary Explanations of One ESL Teacher : A Microanalytic Inquiry », Language Learning, vol. 54, $n^{\circ} 1$, p. 79-117.

LE MEUR C., 2012, « Le silence du texte - II. Voix à la théorie ", Fabula Atelier de théorie littéraire. Disponible sur : <http://www.fabula.org/atelier.php?Silence_du_texte>.

LLORCA R., 1985, « Approche rythmique du paysage sonore », Bulletin d'Audiophonologie, $\mathrm{n}^{\circ}$ 100, fasc. 1. 1-2, p. 150-156.

MATTER J.-F., 2006, « La prononciation authentique en langue étrangère : un problème négligé », Revue française de linguistique appliquée, vol. XI, $\mathrm{n}^{\circ} 1$, p. 21-32. Disponible sur : <https:// www.cairn.info/revue-francaise-de-linguistique-appliquee-2006-1-page-21.htm>.

MÉGÉVAND M., 2001, « Le plaisir du texte théâtral », Le français dans le monde - Recherches et applications, $\mathrm{n}^{\circ} 316$, p. 39-40.

MESCHONNIC H., 1982, « Qu'entendez-vous par oralité ? ", Langue française, vol. 56, n 1, p. 6-23. Disponible sur : <https://www.persee.fr/doc/lfr_0023-8368_1982_num_56_1_5145>.

MOURATIDOU E., 2009, « Discours didascalique et praxis critique. Quand le théâtre parle du théâtre et du monde », Interstudia, Langue, discours, société, n 4, p. 137-144. 
NGO MBAÏ P. M., 2011, « La parole camerounaise : transparence et/ou opacité ? », Synergies France, $\mathrm{n}^{\circ}$ 8, p. 133-144.

ORION G. F., 2012, Pronouncing American English : sounds, stress, and intonation, $3^{\mathrm{e}}$ éd., Boston, Heinle Cengage Learning.

PAGE C., 2000, « Le jeu dramatique : de la proposition au choix. Mise au travail des idées de Winnicott dans le champ des activités théâtrales à l'école ", Revue française de pédagogie, vol. 130, $\mathrm{n}^{\circ}$ 1, p. 133-141. Disponible sur : <https://www.persee.fr/doc/ rfp_0556-7807_2000_num_130_1_1058>.

PARISSE L., 2011, « Dramaturgies contemporaines de la parole. Perspectives pour le français langue étrangère », Synergies France, ${ }^{\circ}$ 8, p. 115-123.

PATOCKA J., 1992, Introduction à la phénoménologie de Husserl, trad. par E. Abrams, Grenoble, Jérôme Millon.

PUOZzo I., 2013, « Pédagogie de la créativité : de l'émotion à l'apprentissage », Les Cahiers du CERFEE, $\mathrm{n}^{\circ}$ 33. Disponible sur : <https://journals.openedition.org/edso/174>.

RECANATI F., 1981, Les énoncés performatifs : contribution à la pragmatique, Paris, Éditions de Minuit, coll. « Propositions ».

ROLLAND Y., 2011, Apprendre à prononcer : quels paradigmes en didactique des langues ?, Paris, Belin, coll. « Belin Sup Langues ».

RYNGAERT J.-P., 1996, Le jeu dramatique en milieu scolaire, $3^{\mathrm{e}}$ éd., Paris, De Boeck, coll. « Pratiques pédagogiques ».

\section{ANNEXES}

Yasmina Reza, 1994, Art, Paris, Actes Sud, coll. « Actes Sud - Papiers ».

2 personnages Serge, médecin, qui aime l'art et qui vient d'acheter un tableau et Marc son ami

Chez Serge.

Posée à même le sol, une toile blanche, avec de fins liserés blancs transversaux.

Serge regarde, réjoui, son tableau.

Marc regarde le tableau.

Serge regarde Marc qui regarde le tableau.

Un long temps où tous les sentiments se traduisent sans mot.

MARC : Cher?

SERGE : Deux cent mille.

MARC : Deux cent mille ?...

SERGE : Handtington me le reprend à vingt-deux.

MARC : Qui est-ce ?

SERGE : Handtington ?!

MARC : Connais pas.

SERGE : Handtington! La galerie Handtington !

MARC : La galerie Handtington te le reprend à vingt-deux ?... 
SERGE : Non, pas la galerie. Lui. Handtington lui-même. Pour lui.

MARC : Et pourquoi ce n'est pas Handtington qui l'a acheté ?

SERGE : Parce que tous ces gens ont intérêt à vendre à des particuliers. Il faut que le marché circule.

MARC : Ouais...

SERGE : Alors?

MARC :...

SERGE : Tu n'es pas bien là. Regarde-le d'ici. Tu aperçois les lignes?

MARC : Comment s'appelle le...

SERGE : Peintre. Antrios.

MARC : Connu?

SERGE : Très. Très !

Un temps.

MARC : Serge, tu n'as pas acheté ce tableau deux cent mille francs?

SERGE : Mais mon vieux, c'est le prix. C'est un ANTRIOS !

MARC : Tu n'as pas acheté ce tableau deux cent mille francs!

SERGE : J'étais sûr que tu passerais à côté.

MARC : Tu as acheté cette merde deux cent mille francs ?!

(Abry et al., $2008:$ 48)

\section{RÉSUMÉS}

L'utilisation du support dramatique comme outil pédagogique à la prononciation n'est pas nouvelle, mais son utilisation se limite parfois à une lecture. Nous aborderons les différents contours de ce matériel et apporterons un éclairage sur la nécessité de prendre en compte la multimodalité de la parole pour répondre aux besoins langagiers en langues étrangères. La vocalisation ne se cantonne pas à la récitation juxtaposée d'une suite de phonèmes, mais à une interprétation, à un jeu et donc à une prise de possession du texte d'un tiers. De plus, l'intentionnalité de l'apprenant dans le processus d'enseignement-apprentissage prend une part importante dans celle de l'oralité, car son identité peut être remise en cause.

Since many years, the use of drama as teaching material in pronunciation class is regular, but all the particularities of such stuff are not visibles and limited to a reading exercice. We will expose the variety of functions of the dramatic text we can use in a pedagogical way in foreigner languages. Voicing is not limited to put some phonemes next to another, but needs an interpretation, an act and an assimilation of the dramatic text. The implication of the student is also important in the act of teaching and learning, in such process his/her identity could be hurted.

\section{INDEX}

Mots-clés : prononciation, oralité, voix, « focus on form », pratique dramatique.

Keywords : pronunciation, oral system, voice, « focus on form », acting. 
AUTEUR

FANNY AUZÉAU

Sorbonne Université/SIAL - FLE 